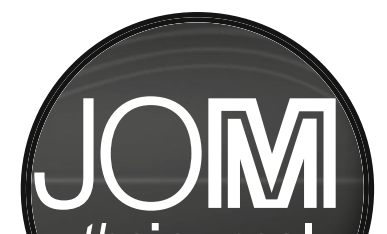
thejournal

\title{
2017 editorial calendar
}

\section{TMS Members:}

Log in to tms.org

for free electronic access to current and archived articles published in JOM, Journal of Electronic Materials, Metallurgical and Materials Transactions $A, B$, and $E$, Integrating Materials and Manufacturing Innovation, and the Journal of Sustainable Metallurgy.

\section{November 2017}

Theme: High-Entropy Alloys

Progress in High-Entropy Alloys

- Beyond Indentation Hardness and Modulus: Recent Advances in Nanoindentation Techniques

- Nanostruc 2016

Nanolaminates

Amorphous Alloys: Liquids and Glasses as Pathways to New Materials

Manuscripts Due: June 1, 2017

\section{December 2017}

Theme: Energy Applications

Field-Intensified Metallurgy

Advanced Materials for Energy Applications

- Thermal Energy Storage and Applications

- Aluminum: Cast Shop and Alloys

Manuscripts Due: July 1, 2017

\section{Coming in June: Progress in Materials Science}

\section{Lightweighting}

- Guest Editor: Will Joost, Pratt \& Whitney, United States

\section{Use of Gases in Pyrometallurgy}

- Guest Editors: Dean Gregurek, Rhi Ag, Austria; Jesse White, Elkem Carbon As, Norway; Zhiwei Peng, Central South University, China

Functional Nanomaterials: Emerging Nanomaterials and Techniques for 3D Architectures

- Guest Editors: Terry Xu, University of North Carolina, United States; and Jung-Kun Lee, University of Pittsburgh, United States 


\section{advisors and guest editors}

\author{
Judy Schneider \\ Additive Manufacturing Bridge \\ Committee \\ Philip Eisenlohr \\ Advanced Characterization, Testing \\ \& Simulation Committee \\ Michael C. Gao \\ Alloy Phases Committee \\ Dmitry Eskin and Pascal Lavoie \\ Aluminum Committee \\ Candan Tamerler and Tolou Shokuhfar \\ Biomaterials Committee \\ Fadi Abdeljawad \\ Chemistry \& Physics of Materials \\ Committee \\ Bala Radhakrishnan \\ Computational Materials Science \\ \& Engineering Committee \\ Vilupajur A. Ravi and Srujan Rokkam \\ Corrosion \& Environmental Effects \\ Committee \\ Christopher Gourlay \\ and Babak Arfaei \\ Electronic Packaging \& Interconnection \\ Materials Committee \\ Tao Wang \\ Energy Committee
}

\author{
Xiaochuan Lu \\ Energy Conversion \& Storage Committee \\ Chantal Sudbrack \\ High Temperature Alloys Committee \\ Takanari Ouchi \\ Hydrometallurgy \& Electrometallurgy \\ Committee \\ Terry Wong \\ ICME Committee \\ Vineet Joshi \\ Magnesium Committee \\ Luana Caron, Orlando Rios, \\ and Ralph Napolitano \\ Magnetic Materials Committee
}

Jian Li, Jiann-Yang Hwang, Bowen Li, and Sergio N. Monteiro

Materials Characterization

Committee

Suveen Mathaudhu

Mechanical Behavior of Materials

Committee

Terry Xu and Jung-Kun Lee

Nanomaterials Committee

Yue Liu and Xinghang Zhang

Nanomechanical Materials Behavior Committee

Ramprashad Prabhakaran

Nuclear Materials Committee

Sudarsanam S. Babu

Phase Transformations Committee
Ma Qian

Powder Materials Committee

Laurentiu Nastac

Process Technology \& Modeling

Committee

Zhiwei Peng, Jesse White, and Dean Gregurek

Pyrometallurgy Committee

Mingming Zhang, Xiaofei Guan, John Howarter, and Randy Kirchain Recycling \& Environmental Technologies Committee

Ravi K. Enneti

Refractory Metals \& Materials Committee

Kester Clarke

Shaping \& Forming Committee

Douglas Matson, Robert Hyers, Mohsen Asle Zaeem

and Amber Genau

Solidification Committee

Sundip Harimkar, Rajeev Gupta, and Kris Darling Surface Engineering Committee

Vikas Tomar and Ritesh Sachan Thin Films \& Interfaces Committee

Peter C. Collins

Titanium Committee

\section{INVITED GUEST EDITORS}

Sanjit Bhowmick

Magnesium-Based Biodegradable Implants

David Bourell

2016 Solid Freeform Fabrication Symposium
Will Joost

Lightweighting

\section{James Njuguna \\ Nanostruc 2016 \\ Vilupanur A. Ravi \\ Archaeomaterials}

Anyone wishing to publish in JOM should follow the guidelines established in the JOM Instructions for Authors, which features detailed information on communication, manuscript preparation, and publication procedures.

The Instructions for Authors are available on the JOM website at jom.tms.org.

For More Information Contact: Telephone: (724) 776-9000 ext. 228 e-mail: jom @tms.org 